\title{
Gravitational waves from galaxy clusters: A new observable effect
}

\author{
Vicent Quilis, José Mâa. Ibáñez \\ and \\ Diego Sáez \\ Departament d'Astronomia i Astrofísica, Universitat de València, E-46100 Burjassot, \\ València, Spain
}

Received — 


\begin{abstract}
A rich galaxy cluster showing strong resemblance with the observed ones is simulated. Cold dark matter spectrum, Gaussian statistics, flat universe, and two components - baryonic gas plus dark matter particles - are considered. We have calculated the gravitational-wave output during the epoch of the fully nonlinear and nonsymmetric cluster evolution. The amplitudes and frequencies of the resulting gravitational waves are estimated. Since frequencies are very small -of the order of $10^{-17} \mathrm{~Hz}$ - a complete pulse cannot be observed during an admissible integration time; nevertheless, it is proved that these waves can produce an interesting secular effect which appears to be observable with current technology.
\end{abstract}

Subject headings: cosmology:theory - gravitation - hydrodynamics - large-scale structure of the universe - methods:numerical - radiation mechanisms 


\section{Introduction}

Any dynamical and nonspherical self-gravitating astrophysical - or cosmological system of mass $\mathrm{M}$ and size $\mathrm{R}$ is a potential source of gravitational waves. The most powerful ones are those combining a very asymmetric shape together with a violent nonstationary evolution.

In present paper we are interested in the gravitational waves radiated by cosmological objects. Flattened structures as superclusters and walls show great departures from spherical symmetry, but they are evolving in the mildly nonlinear regime and, consequently, their evolution is slow. Gravitational waves from these structures will be considered elsewhere. Galaxy clusters evolve in the strongly nonlinear regime and their dynamics is expected to be faster than in the case of larger flattened structures; however, departures from spherical symmetry seem to be small (even if their corresponding initial conditions were fully asymmetric). In this case, high density contrasts - of the order of $10^{3}$ - are reached and, consequently, fully nonlinear numerical simulations are necessary. This paper concerns with the characteristics of the gravitational radiation released during galaxy cluster evolution.

As it is well known, clusters contain dark matter $(\sim 90 \%)$ and a subdominant baryonic component $(\sim 10 \%)$. Since the dynamics and spatial distribution of these components are different, the ratio between the gravitational luminosities coming from these two components is not expected to be constant. Numerical simulations including a baryonic component - gravitationally coupled to the dark one - are necessary to describe the evolution of that ratio.

Taking into account the above discussion, a two components cluster, mimicking the observed ones, has been simulated numerically, paying particular attention to estimate its gravitational luminosity and the features of the radiated gravitational waves. To this 
aim we have used the approach of considering that the sources satisfy the constraints of the so-called nearly Newtonian slow motion. As far as we know, similar calculations have not been performed up to now, probably due to some pessimistic point of view justified by the combination of great numerical difficulties (3D calculations) together with very disappointing small values expected for the gravitational-wave luminosity, the amplitude, et cetera. However, our results have been more encouraging than we could foresee. A new observable effect has been found.

Hereafter, $t$ stands for the cosmological time, $t_{0}$ is the age of the Universe. $\dot{X}$ stands for the derivative of the function $\mathrm{X}$ with respect to the cosmological time. The present value of the Hubble constant is assumed to be $50 \mathrm{Km} \mathrm{s}^{-1} \mathrm{Mpc}^{-1}$. Quantities $c$ and $G$ are the speed of light and the gravitational constant, respectively. Greek (Latin) indices run from 1 to 4 (1 to 3$)$.

\section{Cluster Model}

A flat universe, cold dark matter (CDM), and adiabatic energy density fluctuations are assumed. The corresponding spectrum is normalized by the condition $\sigma_{8}=0.63$. Constrained Gaussian realizations of the density field - in the position space - can be obtained by using the method proposed by Hoffman and Ribak (1991) and improved by Weygaert and Bertschinger (1996). We have used this powerful tool to obtain an initial density field - at redshift $\mathrm{z}=100$ - containing the cluster seed centered in a box. The constraints have been introduced in such a way that, after evolution, our initial overdensity leads to a feasible rich cluster (see Quilis et al. 1998 for details). It is assumed that the $90 \%$ of the matter is CDM and the remaining, the baryonic one, is assumed to be a monoatomic ideal gas. The initial values for the velocity field of both components are identical. No assumptions about the symmetry of the object have been done at all. The resulting 
departures from spherical symmetry correspond to an arbitrary statistical realization of the density field.

The cosmological hydrodynamic equations describing the evolution of the baryonic component (see Peebles, 1980) are solved using a hydro-code based on modern highresolution shock-capturing techniques. This code was described in Quilis et al. (1996). The motion of dark matter particles is studied by means of a standard Particle Mesh code (Hockney and Eastwood 1988). The total gravitational field is computed by solving the Poisson equation -which couples baryons and dark matter- with a multidimensional method based on Fast Fourier Transform (Press et al. 1987). Details about our galaxy cluster simulations are described in Quilis et al. (1998).

At present time, the simulated cluster has: (i) a X-ray luminosity of $\sim 10^{44} \mathrm{erg} / \mathrm{s}$, (ii) a temperature of $\sim 3 \times 10^{7} \mathrm{~K}$, and a total mass inside the Abell radius $(3 \mathrm{Mpc}$ ) of $5.4 \times 10^{14} M_{\odot}$; hence, we are considering a rich cluster having features compatible with present observations (Böeringer, 1991; Peebles, 1994).

\section{Gravitational Radiation}

Our cluster evolves in a flat universe and it is observed with a detector of gravitational waves moving with the cosmological expanding background. This is the most natural reference frame in Cosmology and it is locally Minkowskian at any time. The metric distance from the detector to the cluster, $D(t)$, is proportional to the scale factor $a(t) \propto(1+z)^{-1}$. Its present value is assumed to be $D\left(t_{0}\right)=100 \mathrm{Mpc}$. Gravitons reaching the detector at present time were emitted by the cluster at time $t_{E}$ (emission event $\mathrm{E}$ ). Could we perform our calculations in the Minkowskian space tangent to the Robertson-Walker spacetime at E? The particle point of view is appropriate to answer this question. The emission 
and propagation of gravitons in the real and tangent spaces are now compared: (i) Since graviton emission only depends on the internal dynamics of the system, and it is the same in both cases, the same amount of gravitons with the same energies are emitted, (ii) The background universe as well as the Minkowski empty spacetime are transparent to graviton propagation and, (iii) we have studied the null geodesics in both spacetimes to calculate the times $t_{R W}$ (Robertson-Walker) and $t_{M}$ (Minkowski) elapsed by a graviton in reaching detectors located at the same distances from the cluster. The resulting values of $t_{R W}$ and $t_{M}$ appear to be identical to first order in the parameter $D(t) / 6000$. This parameter is much smaller than unity because the distance $D(t) \leq 100 M p c$ is much smaller than the horizon scale of $6000 \mathrm{Mpc}$. This means that, in both cases, the detectors are receiving signals coming from the same retarded positions of the cluster. After these considerations we can state that our estimates can be performed in the Minkowskian tangent space. This is a consequence of the fact that gravitons are travelling in a region whose size is much smaller than that of the horizon. Only for far clusters observed from distances comparable to that of the horizon $\left(D_{0} \geq 600 \mathrm{Mpc}\right)$, the Minkowskian point of view is not valid and the approach used in this paper must be improved.

Since our calculations can be carried out in the Minkowskian tangent space and galaxy clusters are far of being relativistic in both senses, special relativity, i.e., $v / c \leq 10^{-3}$, and general relativity $r_{g} / R \leq 10^{-4}\left(r_{g}=2 G M / c^{2}\right.$ is the Schwarzschild radius of the object), these clusters can be described by using the so-called slow-motion formalism. The spacetime metric can be linearized in the usual way $\left(g_{\mu \nu}=\eta_{\mu \nu}+h_{\mu \nu}\right)$, the transverse traceless (TT) gauge can be used and, everywhere outside the cluster (Misner, Thorne \& Wheeler, 1973), the spatial components of $h_{\mu \nu}^{T T}$ are

$$
h_{i j}^{T T}=\frac{G}{c^{4}} \frac{2}{D} \ddot{I}_{i j}\left(t-\frac{D}{c}\right)
$$

where $I_{i j}$ are the components of the traceless inertial tensor. The contribution of the baryonic 
fluid, $I_{i j}^{B}$, is $I_{i j}^{B}=\int \rho x_{i} x_{j} d^{3} x-\frac{1}{3} \delta_{i j} \int \rho x^{2} d^{3} x$, where $\delta_{i j}$ is Kronecker's delta, $\rho$ is the baryonic density, and $x$ the physical coordinates. The CDM contribution, $I_{i j}^{D M}$, is given by the following summation extended over all the CDM particles, $I_{i j}^{D M}=\sum_{n_{p}} m_{p}\left(x_{i} x_{j}-\frac{1}{3} \delta_{i j} x^{2}\right)$, $n_{p}$ being the number of CDM particles and $m_{p}$ the mass of each particle. Finally, we compute $I_{i j}=I_{i j}^{B}+I_{i j}^{D M}$. Outside the cluster, the relative motion of two neighbouring particles A and B moving with the cosmological background (ideal detector) is fully described by the quantities $h_{i j}^{T T}$. In TT gauge, there is a system of coordinates attached to $\mathrm{A}$, in which the coordinate variations of the particle B are

$$
X_{B}^{i}(t)-X_{B 0}^{i}=\frac{1}{2} X_{B 0}^{j}\left[h_{i j}^{T T}(t)\right]_{A}
$$

where $X_{B 0}^{i}$ stands for the initial coordinates of the particle $B$ and the quantities $h_{i j}^{T T}$ are calculated at point $A$. From this formula, it follows that oscillations in the $h_{i j}^{T T}$ quantities lead to oscillations in the relative position of particles A and B with related frequencies and amplitudes.

In the slow motion approximation, the gravitational luminosity, $L_{G W}$, is given by the well known formula

$$
L_{G W}=\frac{G}{5 c^{5}} \sum_{i j}^{1,3} \dddot{I}_{i j} \dddot{I}_{i j} .
$$

Since $I_{i j}$ is the addition of two terms, the gravitational luminosity can be decomposed in the following evident manner, $L_{G W}=L_{G W}^{B}+L_{G W}^{D M}+L_{G W}^{C R O S}$. A direct calculation of $L_{G W}$ based on Eq.(3) would be very problematic (Finn and Evans, 1990) as a result of the difficulties coming from the numerical noise inherent to the numerical computation of third order time derivatives. However, as several authors have suggested (see, e.g., Mönchmeyer et al. 1991), the second order time derivatives involved in $\ddot{I}_{i j}$ can be written in terms of the quantities $\ddot{\rho}, \dot{\rho}, \ddot{x}$ and, $\dot{x}$, which, in its turn, can be expressed in terms of related variables, taken advantage of the corresponding system of equations governing the motion of baryonic and CDM components. 


\section{Results}

Figure 1 shows our numerical estimates of $L_{G W}^{D M}$ (crosses), $L_{G W}^{B}$ (triangles) and $L_{G W}$ (piecewise continuous line) versus quantity $(1+z)^{-1}$. At redshifts between 30 and 25, CDM and baryons have roughly the same velocity profiles and proportional (according to their relative abundances) density fields. As a consequence, the mass is the only relevant parameter and quantity $L_{G W}^{B}$ is smaller than $L_{G W}^{D M}$. Since evolution leads to a CDM configuration more stationary and spherically symmetric than the baryonic one, the luminosity $L_{G W}^{B}$ becomes dominant at $z \sim 9$. The total luminosity $L_{G W}$ is a little greater than $10^{36} \mathrm{erg} / \mathrm{s}$ in the redshift intervals $(25,30)$ and $(3,7)$. The fact that luminosities appear to be similar in both periods can be easily understood taking into account that, in the first interval, deviations from spherical symmetry are greater than in the second one, while dynamics is more violent between redshifts 7 and 3. The luminosity, assumed constant, of a source radiating a total energy of $E_{G W} \sim 0.15 M_{\odot} c^{2}$ during the age of the Universe is $\sim 10^{36} \mathrm{erg} / \mathrm{s}$. This means that the efficiency of the gravitational-wave emission from the cluster is $\varepsilon=\frac{E_{G W}}{M c^{2}} \sim 10^{-16}$. Therefore, we cannot expect any energetically significant gravitational-wave background generated by galaxy cluster evolution.

Quantities $h_{+}=h_{x x}^{T T}$ and $h_{\times}=h_{x y}^{T T}$ have been evaluated along the world line of an observer moving with the background who carries the ideal detector of Section 3. The z-axis has an arbitrary direction and the gravitational wave travels along this axis. Quantities $h_{+}$ and $h_{\times}$oscillate with time having very large characteristic periods. Taking into account that, according to Eq. (四), quantities $h_{+}$and $h_{\times}$are proportional to $D^{-1} \propto 1+z$, one easily concludes that the variations of amplitudes are proportional to $1+z$ (effect of background expansion). Hence, the oscillations of the quantities $h_{+} /(1+z)$ and $h_{\times} /(1+z)$ also have varying amplitudes, but the variations of these new amplitudes only depend on the cluster evolution itself. These quantities are plotted, as a function of $t$, in Fig. 2. 
It is noticeable that the luminosities and amplitudes given in Figs. 1 and 2 are compatible with rough estimates based on formulae from other astrophysical scenarios; in fact, according to Shapiro \& Teukolsky (1983) the gravitational-wave luminosity of a self-gravitating system of mass $\mathrm{M}$, size $\mathrm{R}$ and typical velocity $v$ is

$$
L_{G W} \approx L_{0}\left(\frac{r_{g}}{R}\right)^{2}\left(\frac{v}{c}\right)^{6}
$$

being $L_{0}=c^{5} / G \approx 3.63 \times 10^{59} \mathrm{erg} / \mathrm{sec}$. For our simulated cluster is $\left(\frac{r_{g}}{R}\right) \approx 10^{-4},\left(\frac{v}{c}\right) \approx 2 \times 10^{-3}$, and Eq. (何) leads to $L_{G W} \approx 2 \times 10^{35} \mathrm{erg} / \mathrm{sec}$. Moreover, the relative strain or amplitude of the gravitational wave signal coming from a collapsing object at a distance $D$ of the terrestrial detector - in a form well-adapted to our cosmological scenario - is (Shapiro \& Teukolsky, 1983)

$$
h \approx 3 \times 10^{-11}\left(\frac{\varepsilon}{10^{-15}}\right)^{2 / 7}\left(\frac{M}{10^{15} M_{\odot}}\right)\left(\frac{D}{100 M p c}\right)^{-1} .
$$

For our simulated cluster $\left(\varepsilon \approx 10^{-16}\right)$, Eq. (5) leads to $h \approx 0.8 \times 10^{-11}$. Finally, Schutz (1997) gives the following alternative formula:

$$
h \approx 5 \times 10^{-11}\left(\frac{E_{G W}}{10 M_{\odot} c^{2}}\right)^{1 / 2}\left(\frac{f_{G W}}{10^{-17} H z}\right)^{-1 / 2}\left(\frac{D}{150 M p c}\right)^{-1}
$$

where $f_{G W}$ is the frequency of the wave. In the case of our cluster, the characteristic dynamical time is $\approx t_{0}^{-1}$. This gives $f_{G W} \approx 10^{-17} H z$. Furthermore, we have $E_{G W} \approx 0.15$, and $D=100 M p c$. Taking into account all these data, Eq. (6) leads to $h \approx 10^{-11}$.

After describing Figs. 1 and 2 and analyzing their contents and consistency, let us focus our attention on an important consequence. The characteristic periods of the waves coming from the cluster are larger than $10^{9}$ years and, obviously, it is not possible to detect any complete pulse. However, we are going to show that the oscillatory fields $h_{+}$and $h_{\times}$ produce an effect which could be observable - during an admissible integration time - with a standard detector like the one described in Eq. (2). In fact, during the time interval 
$\Delta t$, the distance between two particles $\mathrm{A}$ and $\mathrm{B}$ aligned with the $x$ or $y$ axis undergoes a relative variation $\frac{1}{2}\left[d h_{+} / d t\right]_{0} \Delta t$ and, when the particles are aligned with the directions forming angles of $45^{\circ}$ with these axis, their relative distance have changed in a quantity given by $\frac{1}{2}\left[d h_{\times} / d t\right]_{0} \Delta t$. The above derivatives - to be performed at present time - can be estimated from the data displayed in the curves of Fig. 2. The resulting values are $\left[d h_{+} / d t\right]_{0}=-1.26 \times 10^{-20} y^{-1}$ and $\left[d h_{\times} / d t\right]_{0}=-4.65 \times 10^{-21} y^{-1}$. In brief, our simulated

cluster produces changes in the relative distance of the order of $10^{-22}$ - detectable with current technology - in a short period of four days. This variation in the relative distance is an effect which would last - at the same rate - for many years; in this sense, we can speak about a secular effect. The relative separation distance would vary regularly reaching the order of $10^{-19}$ after one decade.

\section{Conclusions and Discussion}

We have proved that gravitational radiation from clusters does not contribute significantly to the density parameter.

A new effect produced by the gravitational waves emitted by a cluster has been described. A cluster with the features of the observed ones has been simulated in the standard CDM model. In other cosmogonies, the effect produced by clusters having these features is expected to be comparable. This is because the clusters producing the secular effect are located near the observer and, consequently, their evolution -well inside the horizon- is dominated by internal interactions. Simulations support this idea, see, e.g., Huss, Jain \& Steinmetz (1998), who claim that the differences between individual cluster realizations of a given model are more important than the differences within differing scenarios for a given realization. However, the properties of the spatial distribution of clusters depend on the chosen scenario strongly. A few words about detection and future 
work are worthwhile.

We could take advantage of the fact that the secular effect can be observed for many years. In order to do that, this effect should be measured during periods greater than the characteristic time of any time varying gravitational field acting on the detector. Thus, the pulses produced by these fields could be seen as local perturbations of the secular effect.

If the detector is pointing towards a certain direction, it is not receiving gravitational waves from an unique cluster; hence, the calculation of the total effect produced by a set of clusters is important. For the sake of briefness, we present a rough estimate based on idealized cluster distributions within a sphere of radius $R_{s}$. All the clusters are identical to that of this paper. The distance between any pair of clusters is constrained to be larger than a fixed distance $L$. No cluster correlations are considered. Given an observation direction, the contribution of each cluster to the relative variation of the distance between particles $A$ and $B$ (detector) is assumed to be $\frac{1}{2} \Psi \Delta t$ with $\Psi=1.26 \times 10^{-20}\left(\frac{100}{D}\right) \kappa \cos \alpha y r^{-1}$, where $\alpha$ is the angle formed by the line of sight of the cluster and the observation direction. Since clusters do not radiate in a coherent way, their contributions to $\Psi$ are not correlated. This fact is simulated by using the random number $\kappa$ uniformly distributed in the interval $(-1,1)$. The factor (100/D) has been introduced because the effect of a given cluster decreases with distance as $D^{-1}$ (see Eq. (1)). After superimposing the contribution of all the clusters, the mean $\mathcal{M}$ and the standard deviation $\sigma$ have been calculated from the $\Psi$ values corresponding to many observation directions. For each pair $\left(R_{s}, L\right)$, various simulations have been done. The mean $\mathcal{M}$ changes from one to another, but $\sigma$ is a very stable quantity. For the pairs $\left(R_{s}=600 \mathrm{Mpc}, L=50 \mathrm{Mpc}\right),\left(R_{s}=600 \mathrm{Mpc}, L=100 \mathrm{Mpc}\right)$, $\left(R_{s}=500 \mathrm{Mpc}, L=50 \mathrm{Mpc}\right)$, we have found $\sigma=7.6 \times 10^{-20}, \sigma=2.7 \times 10^{-20}$ and, $\sigma=7.0 \times 10^{-20}$, respectively. From these data one concludes that, for $R_{s} \geq 500$, quantity $\sigma$ depends on $R_{s}$ weakly. This fact suggests that the use of radius $R_{s}>600$ is not necessary 
because the main part of the effect is produced by nearby clusters. Furthermore, the resulting $\sigma$ values are greater than the value $1.6 \times 10^{-20}$-maximum value assumed for a single cluster and $\mathrm{D}=100$ - even when large separations $(L=100 \mathrm{Mpc})$ are assumed. The large $\sigma$ values given by simulations strongly suggests the feasibility of anisotropy measurements. Improved simulations including cluster correlations should increase the anisotropy. The effect of realistic spatial distributions including correlations and clusters with different masses, sizes, et cetera will be studied elsewhere. Various parameters -as the proportions between baryonic and dark matter, the density parameter, et cetera- would be involved in these simulations. The resulting anisotropy would depend on both cosmological and large scale structure parameters. Comparisons between measurements and predictions of the secular effect and its anisotropies could play a crucial role in Modern Cosmology.

This work has been supported by the Spanish DGES (grants PB96-0797 and PB940973). V. Quilis thanks to the Conselleria d'Educació i Ciència de la Generalitat Valenciana for a fellowship. Calculations were carried out in a SGI O2000 at the Centre de Informática de la Universitat de València. 


\section{REFERENCES}

Böeringer, H. 1991, New Insights into the Universe, eds. Martínez, V.J., Portilla, M. \& Sáez, D. (Springer-Verlag, Berlin)

Finn, L.S. \& Evans, C.R. 1990, ApJ, 351, 588

Hockney, R.W. \& Eastwood, J.W. 1988, Computer simulation using particles (IOP Publishing)

Hoffman, Y. \& Ribak, E. 1991, ApJ, 380, L5

Huss, A., Jain, B. \& Steinmetz, M. 1998, MNRAS, in press, astro-ph/9703014

Misner, C.W., Thorne, K.S. \& Wheeler, J.A. 1973, Gravitation (Freeman, San Francisco)

Mönchmeyer, R., Schäfer, G., Müller, E. \& Kates, R.E. 1991, A\&A, 246,417

Peebles, P.J.E. 1980, The Large-Scale Structure of the Universe (Princeton: Princeton Univ. Press)

Peebles, P.J.E. 1994, Principles of Physical Cosmology (Princeton: Princeton Univ. Press)

Press, H., Flannery, B.P., Teukolsky, S.A. \& Vetterling, W.T. 1987, Numerical Recipies. The art of Scientific Computing (Cambridge University Press)

Quilis, V., Ibáñez, J.Mํ. \& Sáez, D. 1996, ApJ, 469, 11

Quilis, V., Ibáñez, J.Mạ. \& Sáez, D. 1998, ApJ, in press, astro-ph/9803059

Schutz, B.F. 1997, in Les Houches School on Astrophysical Sources of Gravitational Radiation, eds. J.-A. Marck \& J.-P. Lasota (Cambridge University Press)

Shapiro, S.L. \& Teukolsky, S.A. 1983, Black Holes, White Dwarfs and Neutron Stars (John Wiley) 
van den Weygaert, R. \& Bertschinger, E. 1996, MNRAS, 281, 84

This manuscript was prepared with the AAS IATEX macros v4.0. 
Fig. 1.- Gravitational-wave luminosity as a function of redshift $z$. Piecewise continuous line, crosses and triangles correspond to the total $\left(L_{G W}\right)$, dark matter $\left(L_{G W}^{D M}\right)$, and baryonic $\left(L_{G W}^{B}\right)$ luminosities, respectively.

Fig. 2.- Quantities $h_{+} /(1+z)$ and $h_{\times}(1+z)$ as functions of the cosmological time $t$. 


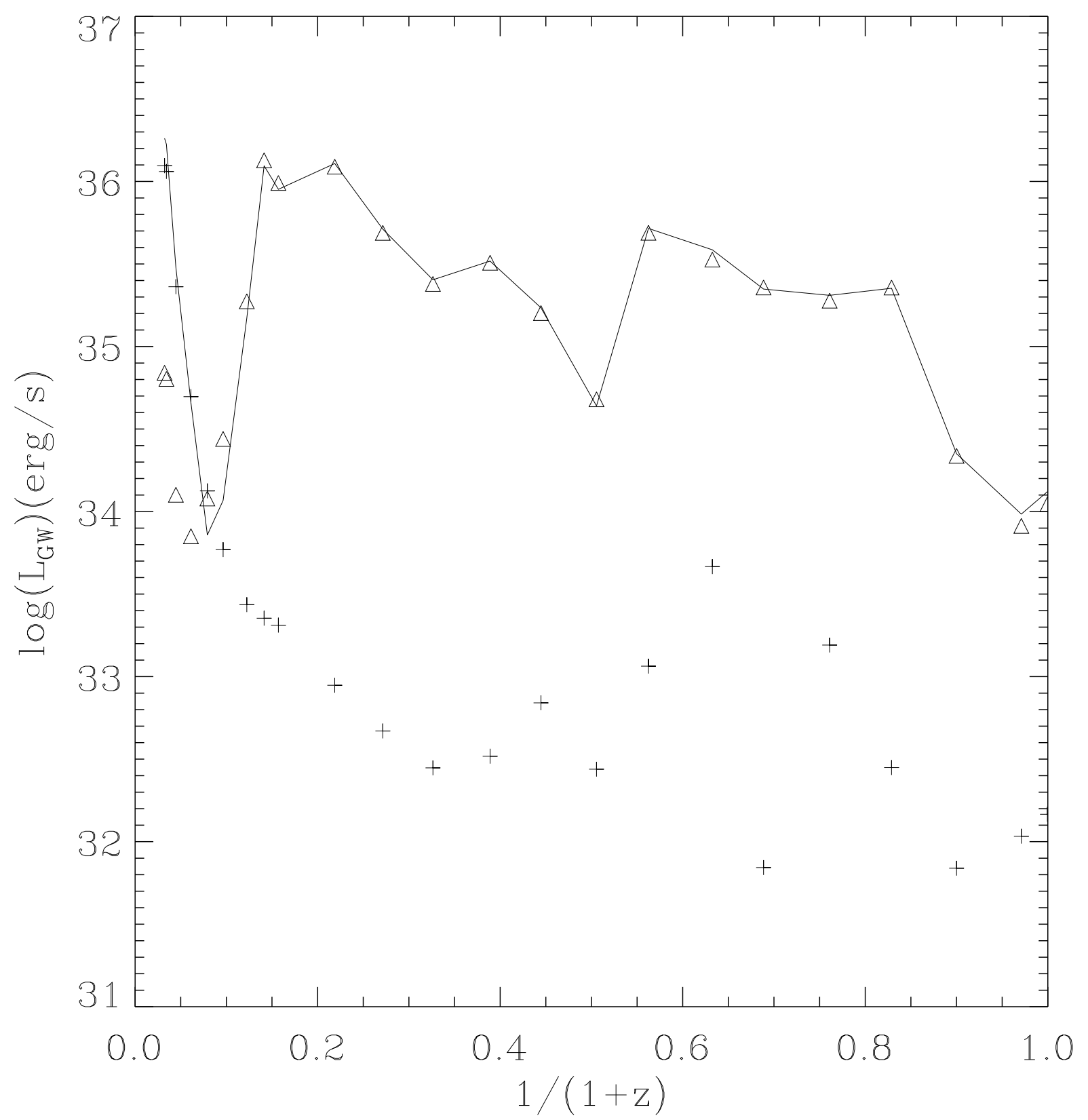




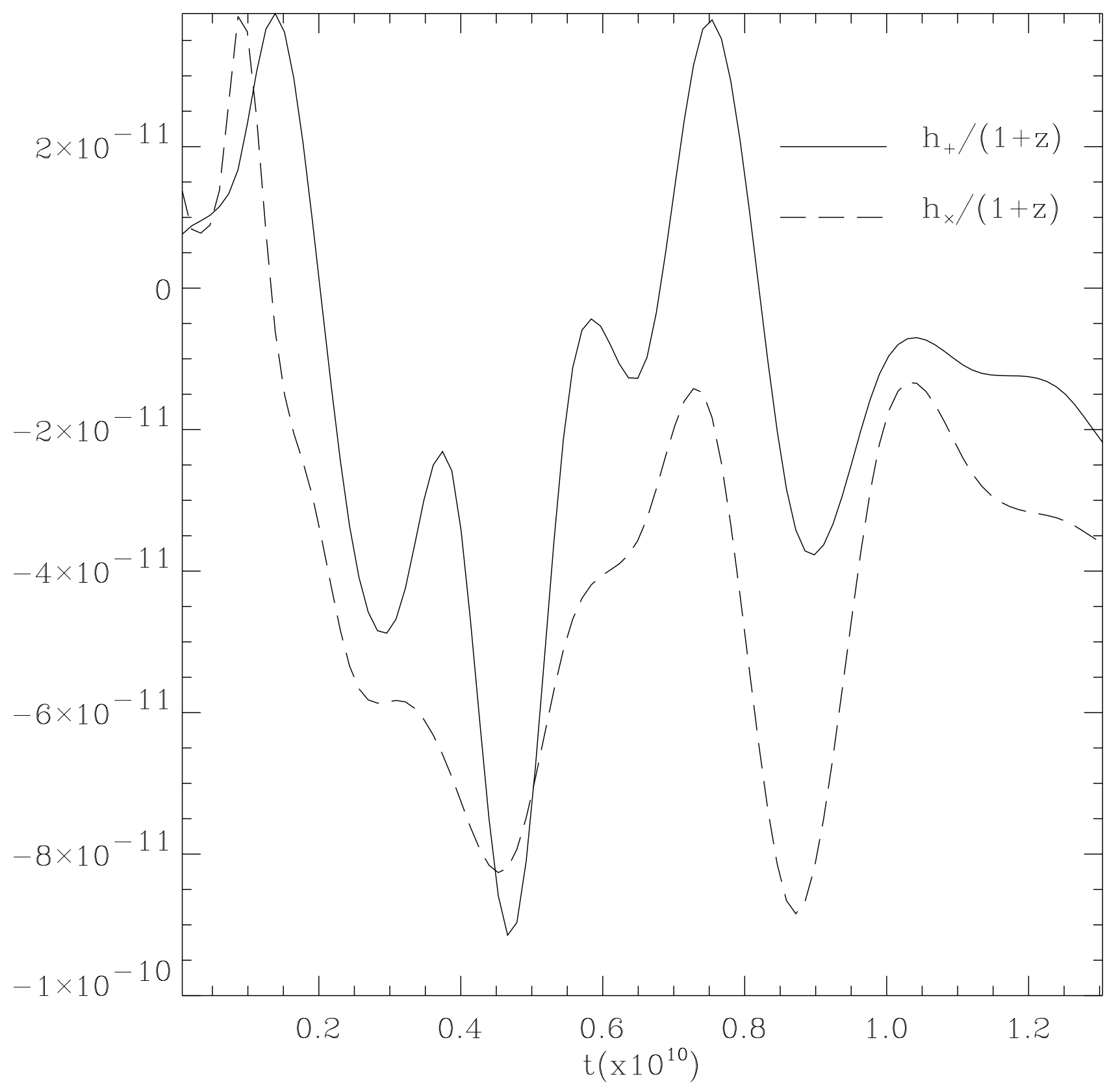

